mit Erfolg und unter allseitiger Anerkennung geführt. Das gewaltige Krankenmaterial, das Leipzig bot, wurde für den demonstrativen Unterricht in der poliklinischen „Ambulanz" gründlichst ausgenutzt. Durch fünf $z$. T. spezialistisch ausgebildete Assistenten war dafür gesorgt, daß die Studierenden alle unentbehrlichen Untersuchungsmethoden in der Praxis übten und handhaben lernten. Die Apparatur seiner Poliklinik hat Hoffmann sich stets besonders angelegen sein lassen, sie soll nach dem Urteil von Kennern in ihrer Vollständigkeit und darin, daß sie stets auf der Höhe des Modernen gehalten wurde, vorbildlich gewesen sein.

Doch wäre es $z u$ wenig in Hoffmanns Art gewesen, wenn er unter der Sorge für das Moderne das Altbewährte hätte Schaden leiden lassen: die Revierbehandlung der Kranken hat er allen Schwierigkeiten zum Trotz seiner Leipziger Poliklinik als integrierenden Bestandteil erhalten.

Dem Polikliniker ist es nicht so leicht gemacht wie dem Kli niker, eine "Schule" zu bilden. Auch hierin hat Hoffmanns Poli klinik mehr wie andere geleistet, aus seinem Assistentenstab sind bekannte medizinische Lehrer und Krankenhausvorstände hervor gegangen: ich nenne nur WINDSCHEID, FRIEDRICH (Otiater in Kiel) v. CrIEgerN. Zu seinem 7o. Geburtstage konnten ihm seine Schüler eine stattliche Festschrift überreichen.

Von HofFMANNS Werk, wie es in wissenschaftlichen Veröffentlichungen vorliegt, habe ich seiner experimentellen Arbeiten bereits gedacht, von späteren größeren Werken sind außer den zwei soeben genannten herauszuheben die Vorlesungen über allgemeine Therapie und das Lehrbuch der Konstitutionskrankheiten.

Die Vorlesungen über allgemeine Therapie stammen aus Dorpat. Sie haben sich einen bleibenden Platz in der Geschichte der Medizin dadurch erworben, daß HofruanN hier zuerst bewuBt und klar die "Schonung und Übung des erkrankten Organs" als die einfachste und erfolgreichste Grundlage jeder vernünftigen Therapie hinstellte und eindringlich lehrte. Diese "Vorlesungen" haben auf weite ärztliche Kreise klärend und anregend gewirkt. Das Lehrbuch der Konstitutionskrankheiten fällt (I893) in eine Zeit die gerade dieser Lehre eine so überraschende Entwicklung brachte - man denke an die endokrinen Drüsen - daß jedem Versuche, sie zu fixieren und darzustellèn, nur eine ephemere Bedeutung beschieden sein konnte. Wertvoll aber ist auch dieses Werk durch den Geist den es atmet; HoFFMANN selbst verlautbart ihn in seinem
Vorwort: ,DasWerk", so sagt er, ,hält sich fern von weitschweifenden Diskussionen, theoretischen Spekulationen, es lehrt die Quellen finden, aus denen unsere Kenntnisse stammen; es soll ermöglichen, daß ein jeder sich Rat erholen kann, so eingehend ihn unsere Wissenschaft zur Verfügung hat. Denn das Lesen der Handbücher allein gibt keinen Begriff von den Schätzen, die unsere Literatur birgt; jeder Arzt sollte die Fähigkeit haben, sich Rechenschaft zu geben von dem, was wir wissen und nicht wissen, um sich unabhängig $2 u$ fühlen von dem, was sein neuester Journalartikel bringt. Möge es gelingen, diese Notwendigkeit dem Bewußtsein der jüngeren Generation wieder näherzubringen. Deñ allzusehr überwuchert die Neigung, unsere Medizin als eine Kunst allein zu betrachten." Wenn HoFFMANN sich mit diesen Worten direkt gegen die wendet, welche die Heilkunde als "Kunst allein betrachten", so gewinnt dies besondere Bedeutung dadurch, daß er selbst viel von der Künstlernatur hatte. Von seinen mannigfachen Talenten haben musikalische und dichterische Begabung keine geringe Rolle in seinem Leben bis in sein Greisenalter gespielt: sein letzter Brief, der, wie sein Sohn schreibt, noch Vollkraft atmet, ist in diesem August aus Bayreuth geschrieben, wo er - erst spät zu dessen Muse bekehrt - Wagners Kunst in voller Lebensfreude genoß. Aber weit über diese Empfänglichkeit und diese Talente hinaus zeigte sein reiches, lebhaftes Innenleben die Künstlerseele; in wahrhaft dichterischer Gestaltungskraft lieB er die Natur mit ihrem ganzen Geschehen in Seele und Welt auf sich wirken, alles lebte und klang an in ihm bis zum Baumblatt und zum Grashalm. Es ist ausgeschlossen, daß HOFFMANN bei solcher Veranlagung hätte die Rolle unterschätzen können, welche ernste Intuition in der Heilkunde spielt, er wußte, was er wollte, wenn er hier vor der Phrase vom Künstlertum des Arztes warnt!

HoFfmanns Begabung war in der Tat eine intuitive, nicht eine dialektisch angelegte! Daher seine bis zur entschiedenen Abneigung gehende Zurückhaltung gegen öffentliches Sprechen: es lag ihm kaum noch, weil ihm zu wenig daran lag. Dem, der ihm nahestand, mochte es wohl einmal scheinen als trüge an seiner Zurückhaltung jeder Öffentlichkeit gegenüber die Furcht schuld vor Profanation der Heiligtümer des eigenen Innenlebens. Weit ging solche Furcht aber nicht, hierzu war er sich seiner Persönlichkeit und dieser ihrer Heiligtümer zu sicher.

B. Naunyn, Baden-Baden.

\title{
ALFRED SCHNABEL $\dagger$.
}

Am I2. Dezember I924 erlag A. Schnabel (geb. am 5. August I89r in Wien) einer septischen, von Hautfurunkeln ausgehenden Erkrankung. Nur 6 Jahre hat das Schicksal dem begabten jungen Forscher gegönnt, um sich in das von ihm gewählte Fach (Ätiologie und Immunitätsverhältnisse der Infektionen) einzuarbeiten. Aus dem Feldzug I9I 8 zurückgekehrt, trat er in das baktetiologische Laboratorium des Militärsanitätskomitees in Wien ein, kam rgIg als Assistent an das bygienische Institut in Basel, wurde abe schon I92I zum Abteilungsleiter am Robert Koch-Institut ernannt und erhielt I924 den Titel eines Professors. Schon dieser ungewöhnliche Aufstieg zu äuBerer Anerkennung legt dafür Zeugnis ab, wie rasch Schnabel die Phasen des Anfängers zu über winden und sich einen geachteten Platz in der wissenschaftlichen Welt zu erobern wuBte. Wer Gelegenheit hatte, die Ent- wicklung dieses Mannes zu verfolgen, wird seine Publikationen (Ưber die Verteilung der Chinaalkaloide im Organismus, über Bakterienüberempfindlichkeit, über das Twort-d'Herellesche Phänomen, über die Encephalitis epidemica) nicht nur vom absoluten Standpunkt aus als achtunggebietende Leistungen bewerten, sondern in ihnen verheißungsvolle Ansätze zu bedeutenderem Schaffen erblicken, zu dem ScHNABEL durch seine kritische Veranlagung, durch geistvolles Erfassen wichtiger Zusammenhänge und durch eine außerordentliche Arbeitskraft befäligt war. Er hat seine weitausschauenden Pläne, die ihn bis in die letzten Tage erfüllten, nicht verwirklichen können; die Wissenschaft hat durch ScHNaBELs Tod - ich darf das als sein Lehrer behaupten - einen großen Verlust erlitten.

R. DOERr, Basel.

\section{TAGESGESCHICHTE.}

Gesundheitsnachrichten. In Preußen sind in der Zeit vom 7. September bis zum 4. Oktober I924 nachstehende Erkrankungen (Todesfälle) an übertragbaren Krankheiten amtlich gemeldet worden : Diphtherie I864 (87), übertragbare Genickstarre 32 (I8), Kindbettfieber 343 (89), spinale Kinderlähmung 6o (I), Körnerkrankheit I57, Lungen- und Kehlkopftuberkulose 3634 (I797), Rotz I (I) übertragbare Ruhr 467 (36), Scharlach 223I (27), Tollwut I (I), Unterleibstyphus I677 (1 25), Fleisch-, Fisch- und Wurstvergiftung I 58 (3), BiBverletzungen durch tolle oder tollwutverdächtige Tiere I 26 .

Der Epidemiebericht Nr. 73 des Hygieneausschusses im Völkerbund meldet über die Seucheñbewegung im Oktober und November 1924 keine besonderen Änderungen oder neuen Vorgänge. Die Pesterkrankungen waren bis auf Britisch-Indien vereinzelt; zum Ausbruch der Lungenpest in Los Angeles wird mitgeteilt, daß bis zum 24. November 4o Pesterkrankungen mit 34 Todesfällen vorkamen, davon nur 5 Fälle von Bubonen; das Auffinden pestinfizierter Nager hat energische $\mathrm{Ab}$ wehrmaßnahmen veranlaßt; die Krankheit selbst scheint zur Zeit erloschen. Cholera kam nur in Britisch-Indien vor. Fleckfieber und Recurrens traten überall sehr spärlich auf. Die Zahl der Pocken- erkrankungen war überall in Europa sehr gering bis auf England, wo sie noch immer zunehmen (318 Erkrankungen im November gegen 223 in den vorangegangenen 4 Wochen). Die Zunahme in den vereinigten Staaten hält sich in den jahreszeitlichen Schwankungen. Unterleibstyphus zeigte vielfach geringe Zunahme, zumal in den baltischen Staaten, am stärksten in Polen und Deutschland, wo die erhöhten Zahlen des Vorjahres noch überstiegen wurden. Ruhr hat in den meisten Ländern gegenüber dem Vorjahr abgenommen, in Deutschland auf die Hälfte der Zahlen des Vorjahres; nur in Polen sind die Zahlen stärker angewachsen. Grippe trat nicht auf; Encephalitis lethargica in Skandinavien vereinzelt, die Epidemie in England besteht weiter in gleicher Höhe fort. Poliomyelitis nahm in Skandinavien weiter ab. Scharlach hat in West- und Mitteleuropa überall nicht unerheblich zugenommen, während der Südosten niedrigere Zahlen zeigt; aus RuBland werden größere Epidemien gemeldet, die seit Juli herrschen. Diphtherieerkrankungen blieben weiter niedrig bis auf England mit einer etwas größeren Steigerung. Die aus dem europäischen Rußland gemeldeten Zahlen für Malaria sind noch immer außerordentlich hoch, für Mai und Juni wurden rund 70o ooo und 500 ooo Erkrankungen gemeldet. 